\title{
BMJ Open SafeFit Trial: virtual clinics to deliver a multimodal intervention to improve psychological and physical well-being in people with cancer. Protocol of a COVID-19 targeted non-randomised phase III trial
}

Chloe Grimmett (D) , ${ }^{1}$ Andrew Bates, ${ }^{2}$ Malcolm West, ${ }^{2,3}$ Samantha Leggett, ${ }^{2}$ Judit Varkonyi-Sepp, ${ }^{2}$ Anna Campbell, ${ }^{4}$ June Davis, ${ }^{5}$ Stephen Wootton, Clare Shaw, ${ }^{8}$ Rachael Barlow, ${ }^{9}$ Joanna Ashcroft, ${ }^{10}$ Andrew Scott (1) , ${ }^{11}$ Helen Moyes, ${ }^{2}$ Lesley Hawkins, ${ }^{12}$ Denny Z H Levett, ${ }^{2,13}$ Fran Williams, ${ }^{14}$ Michael P W Grocott, ${ }^{2,13}$ Sandy Jack $^{2}$

To cite: Grimmett C, Bates A, West M, et al. SafeFit Trial: virtual clinics to deliver a multimodal intervention to improve psychological and physical well-being in people with cancer. Protocol of a COVID-19 targeted non-randomised phase III trial. BMJ Open 2021;11:e048175. doi:10.1136/ bmjopen-2020-048175

- Prepublication history and additional supplemental material for this paper are available online. To view these files, please visit the journal online (http://dx.doi.org/10.1136/ bmjopen-2020-048175)

MPWG and SJ are joint senior authors.

Received 18 December 2020 Accepted 23 July 2021

Check for updates

(C) Author(s) (or their employer(s)) 2021. Re-use permitted under CC BY-NC. No commercial re-use. See rights and permissions. Published by BMJ.

For numbered affiliations see end of article.

Correspondence to

Dr Chloe Grimmett;

C.Grimmett@soton.ac.uk

\section{ABSTRACT}

Introduction The impact of the COVID-19 pandemic (caused by the SARS-CoV-2 virus) on individuals with cancer has been profound. It has led to increased anxiety, distress and deconditioning due to reduced physical activity. We aim to investigate whether SafeFit, a multimodal intervention of physical activity, nutrition and psychological support delivered virtually by cancer exercise specialists (CES), can improve physical and emotional functionings during the COVID-19 pandemic. Methods and analysis A phase III non-randomised intervention trial, target recruitment of 1050 adults with suspected or confirmed diagnosis of cancer. All recruited participants will receive the multimodal intervention delivered by CES for 6 months. Sessions will be delivered 1-to-1 using telephone/video conferencing consultations. CES will work with each participant to devise a personalised programme of (1) physical activity, (2) basic dietary advice and (3) psychological support, all underpinned by behaviour change support.

Primary outcome Physical and emotional functioning as measured by the European Organisation for Research and Treatment of Cancer-Quality of Life Questionnaire (EORTC-QLQ-C30). Secondary outcomes: overall quality of life measured by EORTC-QLQ-C30 and EQ-5D-5L, health economics, patient activation, self-efficacy to self-manage chronic disease, distress, impact of COVID-19 on emotional functioning, self-reported physical activity, functional capacity and nutrition. Adherence to the intervention will also be measured and a process evaluation conducted. Ethics and dissemination Ethical approval was obtained from the Health Research Authority (reference number 20/NW/0254). Results of this trial will be disseminated through publication of peer-reviewed articles, presentations at scientific conferences, and to the public and people with cancer in collaboration with our patient and public involvement representatives and partners. Trial registration number NCT04425616.

\section{Strengths and limitations of this study}

- The SafeFit Trial will evaluate a novel approach to delivering multimodal exercise, nutrition and psychological support to people with cancer safely during and beyond the COVID-19 pandemic.

- The intervention will be delivered by cancer exercise specialists who have been upskilled using a bespoke training package, including nutrition, psychological support and healthy conversation skills.

- The intervention, underpinned by evidence-based behaviour change techniques, seeks to empower participants to develop new behaviours that can be sustained for the long term.

- Limitations of the trial include absence of a control group and reliance on self-report measures to evaluate behaviour change.

\section{INTRODUCTION}

The COVID-19 pandemic, caused by the SARS-CoV-2 virus, has led to reprioritising of clinical care, and the impact on individuals with a cancer diagnosis has been profound. Treatments and follow-up care have been severely disrupted, affecting 650000 people with cancer in the UK alone, and many supportive services have also been postponed. ${ }^{1}$ ${ }^{2}$ Moreover, once infected with SARS-CoV-2, people with cancer experience significantly worse clinical outcomes. ${ }^{3}$ Although not all people living with and beyond cancer are now advised to shield, many remain fearful of leaving their homes due to the risks of contracting the virus and the consequences of COVID-19. ${ }^{1}$ 
For many people with cancer, the pandemic has resulted in deconditioning due to social isolation, reduced physical activity and changes to eating habits that limit their ability to consume sufficient energy and nutrients to meet their needs. Cancer is typically a disease of older adults who are at particular risk of pulmonary complications as a result of COVID-19, which will likely be exacerbated by reduced cardiopulmonary fitness associated with such reductions in activity levels. Furthermore, smoking, poor nutrition and obesity are independent risk factors for developing cancer, which concurrently increase vulnerability to severe COVID-19. ${ }^{4}$

Good nutrition and regular physical activity have proven to be effective at addressing a variety of disease and treatment-related consequences of cancer, and optimising physical fitness is also likely to decrease morbidity and mortality associated with COVID- $19 .^{5}$ Thus, supporting this population to maximise engagement in physical activity and to improve nutritional status is imperative.

Supporting psychological well-being is also vital for people with cancer. Higher levels of anxiety and depression are associated with poor quality of life and physiological outcomes both early in the treatment pathway and in patients who have completed treatment. ${ }^{6-8}$ Many people with cancer will continue to experience distress, depression and anxiety months and years after cancer treatment completion. These issues are exacerbated by the COVID-19 pandemic through reduced access to informal social support networks and formal psychological support services. Macmillan Cancer Support reported in June 2020 that over 27000 people with cancer in the UK have experienced panic or anxiety attacks because of the COVID-19 pandemic. ${ }^{9}$

The SafeFit Trial, as described in this paper, was conceived when our research team was forced to pause recruitment to the Wessex-Fit-4-Cancer Surgery Trial, ${ }^{10}$ a multimodal prehabilitation trial delivered in community settings. We wanted to develop a new programme to support patients throughout and beyond the COVID-19 pandemic. The multimodal structure of the intervention is informed by the recent Macmillan, Royal College of Anaesthetists and National Institute of Health Cancer and Nutrition Collaboration, Research Principles and Guidance for Prehabilitation within the Management and Support of People with Cancer. ${ }^{11}$ The guidance advocates for a multimodal approach encompassing exercise, nutrition and psychological support in order to optimise patients with cancer prior to treatment, increasing their resilience to withstand cancer therapies and hastening their recovery.

It is now accepted that people with cancer require 'end-to-end' pathway support, at the point of diagnosis, throughout treatment and recovery. The SafeFit Trial adopted the multimodal prehabilitation model for universal provision of support with patients recruited at any point in the treatment and recovery pathway. People with cancer are increasingly turning to remote support services, and distanced and home-based interventions have been shown to be effective in supporting dietary and physical activity behaviour change. ${ }^{12}$ However, evidence suggests that inclusion of a supervised component increases intervention adherence ${ }^{13}$ and longer-term maintenance of physical activity behaviour change. ${ }^{14}$

Considerable research has explored the most effective 'ingredients' of a behaviour change intervention in cancer populations to improve engagement and adherence to such interventions as well as to promote longerterm behaviour change. A recent Cochrane review supports the use of goal setting, setting of graded tasks and instruction on how to perform behaviour to maximise intervention adherence. ${ }^{13}$ Additionally, action planning and social support are associated with maintenance of behaviour change. ${ }^{14}$ Furthermore, there is growing evidence of the role of self-efficacy-a person's belief in their ability to perform a given task-in supporting behaviour change with evidence that self-efficacy is a mediator of exercise behaviour in clinical populations and a predictor of exercise adherence. ${ }^{15}$ The SafeFit Trial is underpinned by behavioural science using evidencebased behaviour change techniques to optimise patient engagement and to support self-management and longterm behaviour change.

The proposed trial explores the impact of SafeFit, a virtually delivered multimodal intervention, on the physical and emotional well-being of people with cancer.

\section{METHODS AND ANALYSIS}

Trial design and setting

The SafeFit Trial is a phase III non-randomised intervention with multimodal components of exercise, nutrition and psychological support delivered remotely by telephone and/or video conferencing.

\section{Trial objectives and outcome measures}

\section{Primary objective}

The primary objective of this study is to investigate the efficacy of SafeFit interventions to improve physical and emotional functioning as measured by change in the European Organisation for Research and Treatment of Cancer-Quality of Life Questionnaire (EORTC QLQC30 $)^{16}$ over the 6 -month intervention. Five items for physical function and four for emotional function are answered using a Likert scale scoring of 1-4 to provide a function score from 0 to 100 . Higher scores represent higher functioning. This subscale has been used in previous interventions in cancer populations and is sensitive to change over time.

\section{Secondary objectives}

The main secondary objectives are to investigate the impact of the SafeFit Trial on:

- Quality of life and cost-effectiveness: overall cancerspecific quality of life and global health status, cognitive and social function, and nine symptom subscales 
will be measured by the EORTC-QLQ-C30. Quality of life will also be measured by the EQ-5D-5L, a standardised instrument developed by the EuroQoL Group for use as a measure of health outcome. Applicable to a wide range of health conditions and treatments, the EQ-5D-5L health questionnaire provides a simple descriptive profile and a single index value for health status. ${ }^{17}$ Resources used to deliver the SafeFit Trial will be measured and valued, and health economic analysis will be conducted using the EQ-5D-5L and the Patient Activation Measure (PAM) (see below further for details).

- Self-efficacy and patient activation: self-efficacy to self-manage chronic disease will be measured by the Self-Efficacy for Managing Chronic Disease Scale, a six-item measure with higher scores indicating greater confidence to manage illness-related problems. ${ }^{18}$ Patient activation will be measured by the PAM. ${ }^{19}$ The PAM is a validated self-report survey. Each survey response is scored and based on the total score between 1 and 100; responders are categorised to four activation levels.

- Psychological distress will be measured using an emotions thermometer, ${ }^{20}$ a simple rapid modular visual analogue screening tool for detection and monitoring of emotional disorders in clinical practice. Four emotional domains (distress, anxiety, depression and anger) are measured using a Visual Analogue Scale (0-10) and one outcome domain-need for help. ${ }^{21}$ The impact of COVID-19 on psychological functioning will be measured by the Impact of Events Scale. ${ }^{22}$ No validated measure is available to measure the impact of COVID-19 on physical function.

- Behaviour change: self-reported physical activity will be measured using the modified Godin Leisure Time Exercise Questionnaire. ${ }^{23}$ This is widely used in the exercise oncology literature and has been validated against objective activity monitoring and measures of physical fitness. ${ }^{24}$ Diet will be measured using the World Cancer Research Fund (WCRF) modified HealthCheck tool,${ }^{25}$ which examines intake of fruits, vegetables, whole grains, red and processed meats, processed foods high in fat and sugar, sugary drinks and alcoholic beverages.

- Self-reported height (baseline only), weight, weight loss and changes in nutritional status will be measured by short-form Patient Generated Subjective Global Assessment. ${ }^{26}{ }^{27}$ Functional capacity will be measured by the Duke Activity Status Index (DASI). The DASI also allows for the calculation of individuals' predicted peak oxygen consumption. ${ }^{28}$

Finally, differences in response to the SafeFit Trial, depending on COVID-19 status (confirmed COVID-19, suspected COVID-19, self-isolation and none), will be explored.

The aforementioned outcomes (except for health economics) will be assessed at 6 months (primary endpoint), in addition to 3 months (mid intervention) and 12 months (postintervention follow-up). A follow-up email/phone call will be made at each time point, if necessary, to maximise data completion.

\section{Exploratory outcomes}

Exploratory outcomes include overall survival (all-cause mortality) at 12 months.

Demographic and clinical data will be collected at baseline, including age, sex, postcode, ethnicity, education, employment status, marital status, living arrangement (who they live with), household accommodation and car ownership. Self-reported clinical data will include date of diagnosis, cancer type and stage, cancer status, treatment/s (current and historical) and comorbidities.

\section{Inclusion/exclusion criteria}

Adults (aged $\geq 18$ years) with suspected or confirmed diagnosis of cancer will be included in this trial. Individuals unable to give informed consent will not be eligible for this trial.

\section{Recruitment and recruitment procedures}

Potential participants will be recruited via self-referral, with the SafeFit Trial advertised through social media, via partner organisations including Macmillan Cancer Support, and through clinical teams and multidisciplinary team meetings.

Potential participants will visit the SafeFit Trial website and complete a Smart Survey to express their interest in the trial. A welcome email will be sent to potential participants, together with a patient information sheet. A member of the trial team will then telephone potential participants to confirm eligibility. During this telephone call, potential participants will complete the following screening to confirm suitability for the trial:

1. The Physical Activity Readiness Questionnaire. ${ }^{29}$ This tool screens participants presenting acute or uncontrolled long-term conditions that would be exacerbated by exercise. ${ }^{30}$

2. COVID-19 status (confirmed COVID-19, suspected COVID-1, self-isolation or none)

3. Nutritional state (problems eating or drinking and unintended weight loss), whether the individuals are receiving nutritional support and if they are under the care of a registered dietitian. Those assessed to be malnourished (body mass index $<18.5$ ) or reporting specific nutritional impact symptoms of dysphagia, diarrhoea or vomiting or receiving artificial nutritional support will not receive the standard nutritional advice element of the trial. Appropriate referrals for nutritional support will be made for those identified as at risk of malnutrition.

4. Psychological distress. Those scoring $\geq 8$ on the distress thermometer are asked additional questions. Those at risk of self-harm will not be recruited to the trial, and appropriate referrals for support will be made.

Participants will be eligible for inclusion to the trial, providing they are safe to receive at least one of the three 


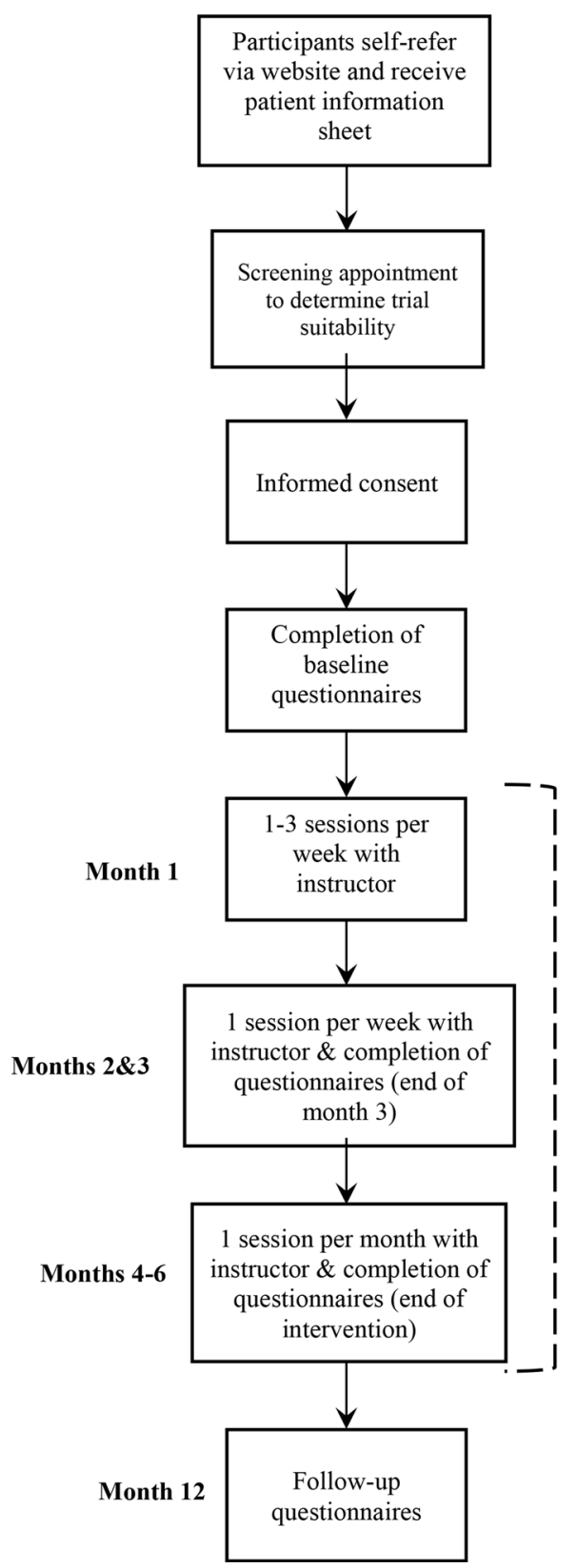

Figure 1 Trial flow.

components. For example, a potential participant who is deemed unsafe to exercise would receive the nutritional and psychological components of the intervention. The exercise element would be introduced if/when it is safe to do so.

All eligible participants will then complete an online consent form and baseline questionnaires. Those not willing or able to complete questionnaires online will be posted paper copies with a return prepaid envelope. Once baseline questionnaires are complete, participants will be matched with aCES. Participants will have the opportunity to complete an electronic Holistic Needs Assessment prior to the telephone call with the trial team. See figure 1 for the trial flow.

\section{Intervention}

The intervention duration will be 6 months. Participants will receive up to three one-to-one sessions per week for 1 month (weeks 1-4), weekly for 2 months (weeks 5-12) and monthly for 3 months (weeks 16, 20 and 24).

\section{Exercise}

Participants will be supported to engage in at least one and up to three exercise sessions per week, including (1) aerobic exercise at a rating of perceived exertion of 11-14 (6-20 scale) accumulating up to $30 \mathrm{~min}$ per session and (2) resistance exercise of $8-10$ different exercises each for $2 \times$ 8-15 repetitions performed in a controlled manner and covering the whole body and range of motion. Resistance exercise should be performed through the full pain-free range of motion covering the whole body with maintenance of good alignment for $10-30 \mathrm{~s}$, with some movements held for a second set of 10-30 s if stiff. Engagement will involve a combination of supervised exercise sessions during the one-to-one sessions (if requested by the participant) and unsupervised home-based sessions.

\section{Psychological support}

The CES will provide psychological support as per level 1 of the Improving Supportive and Palliative Care for Adults with Cancer. ${ }^{31}$ This includes recognising the psychological needs of patients, providing compassionate communication, general psychological support and simple selfmanagement-focused signposting and problem solving.

\section{Nutrition support}

The CES will work with participants to review their diet and eating habits against WCRF recommendations using the modified 'HealthCheck' online tool to identify areas of change as appropriate. ${ }^{25}$ Participants will review their consumption of fruit and vegetables, whole grains, red and processed meat, processed foods high in sugar and fat, processed meats and alcohol intake with the aim to achieve WCRF recommendations for cancer survivors through incremental goal setting. The CES will regularly check for unintended weight loss or changes in gastrointestinal function and/or changes in the ability to eat/ drink and report abnormalities immediately to the trial team.

\section{Behaviour change support}

The CES will receive training in healthy conversation skills. ${ }^{32}$ This will enable them to deliver a client-centred, solution-focused, empowering intervention informed by social cognitive theory. The intervention is aimed at increasing patients' self-efficacy and motivation to adopt behaviour change. The same skillset and delivery modality will be employed to support patients in engaging in the exercise and nutrition components of the intervention as well as adopting strategies to self-manage their psychological well-being. Participants will be provided with a SMARTER (specfici, measurable, achievable, relevant, timely, evaluate and readjust) goal-planning sheet to assist with goal setting and action planning during 
consultations with their CES. The titrated support acts to increase participants' autonomy and support longterm engagement in these new behaviours. See online supplemental appendix A for the list of behaviour change techniques employed as per the taxonomy of behaviour change techniques ${ }^{33}$ and used flexibly within sessions as per the person-centred approach.

\section{Training programme for CES}

All CES will have training in exercise referral and/or additional qualifications in cancer and exercise rehabilitation and will deliver the SafeFit interventions. All CES will also receive a bespoke training package delivered online by the trial team, supported by the clinical team:

1. Health conversation skills-online healthy conversation skills training (online Making Every Contact Count (eMECC) Lite). This training is an online version of the Royal Society for Public Health-accredited MECC Lite Healthy Conversation Skills training. Consistent with the face-to-face training, the online version is highly interactive and experiential. The training equips trainees with skills to create and identify opportunities to hold conversations about health and well-being, to explore the individuals' barriers and facilitators to making change and taking control, to use active listening, and to support individuals to find their own solutions, plan for taking action to implement these solutions, monitor progress and adjust, plan and act as needed.

2. Nutrition-a webinar with accompanying support material will be delivered by an experienced dietitian (CS) to provide training in generic nutritional principles in line with the recommendations from the WCRF and the British Dietetic Association and to identify deterioration in nutritional status. The webinar covers the principles of healthy eating ('eat well' advice), weight management, symptom management, prehabilitation advice before treatment starts, and rehabilitation advice during and after treatment. Links to trusted dietary resources provided on the internet will be made available.

3. Emotional support-a webinar with accompanying supportive materials will be delivered by an experienced clinical psychologist (JA) specialising in oncology. This will focus on communication skills, recognising emotions, active listening and questioning.

\section{Safety during sessions}

It will be the responsibility of the CES to complete a presession screening checklist to monitor condition, medical contacts, medication and COVID-19 status. Exercise will continue, be modified with observation, or stopped and review sought from the treating medical team. In the case of an acute medical event during the exercise session, the CES will advise the participants to call their general practitioner (GP) or 111. If concerned about collapse, the CES will call 999. The CES will ask the participant to repeat the distress thermometer before each session. If the participants score 8 or above for 2 consecutive weeks, they will be encouraged to contact Macmillan Cancer Support helpline and/or their GP. In the case of suicidal or self-harm ideation, the CES will advise contacting Samaritans, SHOUT, GP or 111. If concerned about immediate risk, the CES will call 999. Participants who experience a marked deterioration in their nutritional state (eg, stricture, swallow, inanition and weight loss) will be directed back to their cancer care team. Participants with a confirmed diagnosis or suspicion of COVID-19 will have their exercise intervention paused for 14 days but, symptoms allowing, will be able to receive the other interventions. The exercise intervention will also be paused if anybody in their household is displaying COVID-19 symptoms. Acute events and changes in condition and/ or treatment plan will be reported to the trial team. Cases of immediate physical or mental health concern will be raised with the chief investigator or senior clinician with delegated authority. All other cases will be discussed at a weekly multidisciplinary clinical team review. In case of incomplete information or ongoing investigation, it will be the responsibility of the participant to gain clinical sign-off before resuming trial activity. All adverse and series adverse events will be recorded.

\section{Fidelity checks}

Attendance at each scheduled session will be documented by the CES throughout the trial using session completion logs; these will be regularly reviewed by the trial team. The CES will be offered group supervisions once every 2 weeks to address any concerns during the trial. Approximately $20 \%$ of trainers will have two sessions (initial assessment and one follow-up call) observed (via recording of video or telephone call) and assessed against a bespoke implementation checklist to assess fidelity of intervention delivery, including assessment of competency for delivery of healthy conversation skills.

See Template for Intervention Description and Replication (TIDieR) checklist (online supplemental appendix B) for detailed description of intervention components, training procedures and links to additional resources.

\section{Process evaluation}

A comprehensive process evaluation will enable identification of barriers and facilitators to the implementation of and participation in the SafeFit Trial. It will afford an in-depth understanding of processes, relationships and communications that helped or hindered conduct of the trial.

The process evaluation will assess acceptability of the SafeFit Trial from the perspective of participants and professionals delivering the programme as well as identifying barriers and enablers to engagement with and adherence to the programme. We will also capture data to explain how the intervention worked, who it did and did not work for and why, along with other issues with delivery of the intervention and participant receptivity. Qualitative in-depth semistructured interviews will be conducted with participants enrolled in the trial and professionals 
involved in the delivery of the trial. This will include $n=25$ participants who will be purposively sampled to include a range of age, sex, disease type, time since diagnosis and adherence to scheduled calls. Interviews will focus on the barriers and facilitators to participation in the trial and success (or not) of behaviour change. These data will provide explanatory insight into the findings of the trial.

Interviews will also be conducted with CES delivering the trial as well as administrative personnel coordinating the trial $(n=15)$. The purpose of these interviews is to understand the barriers and facilitators to the delivery of the prescribed interventions as well as views of the usefulness of training received.

Normalisation process theory (NPT) will underpin the conceptual framework that will structure the process evaluation (ie, the interview schedules, findings and their interpretation). NPT provides an explanatory framework to better understand the routine embedding of healthcare interventions in their social contexts, in particular, why some processes seem to lead to a practice becoming sustained over a long term, while others do not. The starting point of NPT are the dynamics associated with the embedding of a practice, that is, what people actually do and how they work together. ${ }^{34}$

\section{Patient and public involvement (PPI)}

People with cancer were consulted at the outset of this trial. We worked closely with four research partners, including individuals living with cancer (who were shielding), caring for someone with cancer and recovering from cancer. They provided suggestions of how potential participants might be reassured of the safety of the trial as well as support they might need to access the virtual intervention. They also reviewed trial questionnaires and patient facing documentation and piloted the selfreferral process. Moreover, they agreed to be members of our steering group and will contribute to the oversight of the trial. In previous trials conducted by our research group, PPI representatives have been invited to speak at conferences and stakeholder events, providing powerful testimonies. We intend to continue this approach with the current trial. The research team will liaise with the PPI involvement lead in University Hospital Southampton's Biomedical Research Centre to identify training and support needs of our research partners throughout the trial.

\section{Statistical analysis plan and sample size calculation}

Preliminary data suggest that approximately $62 \%$ of patients will have a 'good' Ppysical function score of $>83$ at baseline, and $43 \%$ of patients will have a 'good' emotional function score at baseline $(>71)$ as determined by threshold for clinical importance for the EORTCQLQ-C30. ${ }^{35}$ In order to detect an $8 \%$ improvement in the proportion of patients with good physical/emotional function score with $90 \%$ power (alpha $=0.05$ ), 1050 patients will be required (allowing for $20 \%$ drop-out).
Descriptive statistics will be used to summarise baseline demographic and clinical variables. For continuous variables, the mean and SD will be calculated for normally distributed data. If the data are not normally distributed, the median and IQR will be calculated. Categorical or binary variables will be summarised as frequency and percentage of total.

The primary endpoints are EORTC-QLQ-C30 physical and emotional function scales measured at 6 months. The McNemar test will be used to investigate whether there is a difference in the proportion of patients with good physical and emotional fFunction scores at the end of the intervention (6 months) compared with baseline. In order to account for multiple comparisons, the Holm procedure will be used to adjust $\mathrm{p}$ values.

Repeated measures logistic regression will be used to investigate the change over all trial visits (baseline and 3,6 and 12 months) and to adjust for clinically prognostic factors which will include age, gender, cancer type, tumour site and systemic anticancer treatment.

Subgroup analysis will also be performed. The proportion of patients with good physical and emotional function score at 6 months (with CI) will be calculated for each subgroup and will be displayed on a forest plot, along with the $\mathrm{p}$ value for interaction. COVID-19 status (confirmed COVID-19, suspected COVID-19, self-isolation and none), curative versus palliative, chemo/rad versus not, surgery versus not, adherent to intervention versus not (adherence is defined as completing $>70 \%$ of calls with CES), tumour site, baseline quality of life (above 85 vs 85 total EORTC-QLQ-C30 score).

Analysis of secondary endpoints (including but not limited to anxiety, depression, confidence to self-manage chronic disease, physical activity and dietary behaviour change, and Duke activity status) will be performed using the appropriate statistical tests/regression models, depending on the outcome data type (ie, continuous, ordinal and binary), and taking into account the paired nature of the data (before and after intervention). This will be described in a detailed statistical analysis plan.

In the exploratory analysis, the Cox proportional hazards model will be used to investigate the relationship between change in emotional and physical function and mortality within 1 year. The Kaplan-Meier plot will be used to illustrate the survival of different patient groups.

Recruitment commenced in June 2020 with estimated completion date for recruitment and follow-up assessments in August 2022.

\section{Strengths and limitations}

The SafeFit Trial provides a novel approach to deliver exercise, nutrition and psychological support to people with cancer. The virtual method of delivery allows access to this personalised and holistic support from individuals' homes, mitigating any risk of exposure to COVID-19 as well as removing well-established barriers to in-person interventions, including travel and ability to integrate programmes within other life commitments. 
Underpinned by evidence-based behaviour change techniques, it aims to empower participants to establish new behaviours that will be embedded in their everyday lives for the long term. The trial is limited by the lack of comparison group. Measures of behaviour change are self-report and thus may introduce bias.

\section{Ethics and dissemination}

Health Research Authority (HRA) ethical approval was obtained on 20 May 2020 (protocol V.2 date: 13 May 2020), prior to the opening of the trial (reference: 20/ NW/0254). Any protocol modifications will be approved by the HRA before being implemented. Any amendments will be reported on dissemination of the trial. The trial has been registered with ClinicalTrials.gov. The University Hospital Southampton NHS Foundation Trust is the sponsor of this trial. Monitoring and auditing will be conducted in accordance with the sponsor's policies and procedures. An independent data monitoring committee will be convened and will have oversight of trial data management.

Trial results will be disseminated to academics, commissioners, policy makers and the public through several avenues. Journal articles and scientific conferences will be used to disseminate to academic audiences. We will also communicate results to the cancer alliances, charities and through recognised NHS communication systems and social media. The University Hospital Southampton NHS Foundation Trust press office will coordinate press releases of key findings. We will also work in collaboration with our PPI representatives and partners to ensure dissemination to people with cancer.

\section{Data collection, quality and storage}

Data will be collected and stored on password-protected databases by trial personnel who are trained in Good Clinical Practice and General Data Protection Regulations. Confidentiality will be ensured before, during and after the trial and all procedures for handling, storing, destroying, and processing data will be compliant with the Data Protection Act 2018. Patient-reported outcome measures will be completed on paper or using the electronic case report form (ALEA), depending on patient preference. Prior to any statistical analysis, all variables will be checked for the number of missing and impossible values. Impossible values will be defined by clinical opinion. The trial sponsor and chief investigators will have access to the final dataset.

\section{Author affiliations}

${ }^{1}$ School of Health Sciences, University of Southampton, Southampton, UK ${ }^{2}$ NIHR Southampton Biomedical Research Centre, University Hospital Southampton NHS Foundation Trust, Southampton, UK

${ }^{3}$ School of Cancer Sciences, Faculty of Medicine, University of Southampton, Southampton, UK

${ }^{4}$ School of Applied Science, Edinburgh Napier University, Edinburgh, UK

${ }^{5}$ Macmillan Cancer Support, London, UK

${ }^{6}$ School of Human Development and Health, Faculty of Medicine, University of

Southampton, Southampton, UK

${ }^{7}$ NIHR Cancer and Nutrition Collaboration, Southampton, UK
${ }^{8} \mathrm{NIHR}$ Biomedical Research Centre, Royal Marsden NHS Foundation Trust, London, UK

${ }^{9}$ Cardiff and Vale University Health Board, University Hospital of Wales, Cardiff, UK

${ }^{10}$ St George's University Hospitals NHS Foundation Trust, London, UK

${ }^{11}$ School of Sport, Health and Exercise Science, University of Portsmouth, Portsmouth, UK

${ }^{12}$ Critical Care/Anaesthesia and Perioperative Medicine Research Unit, University Hospital Southampton NHS Foundation Trust, Southampton, UK

${ }^{13}$ School of Clinical and Experimental Sciences, Faculty of Medicine, University of

Southampton, Southampton, UK

${ }^{14}$ Wessex Cancer Alliance, Southampton, UK

Twitter Andrew Bates @AndyCBates, Anna Campbell @canrehab and Joanna Ashcroft @Dr_Jo_Ashcroft

Acknowledgements We thank Lisa Young for providing clinical advice and support.

Contributors CG drafted the manuscript. CG, SJ, JD and MPWG made substantial contributions to the conception and design of the trial. JA provided clinical psychology expertise, contributing to the design of the psychological component of the intervention and providing critical intellectual content. SW, CS and RB provided nutrition and dietetic expertise, contributing to the design of the nutritional components of the intervention and providing critical intellectual content. JV-S provided expertise in Healthy Conversation Skills Training, contributing to the design of the behaviour change components of the intervention and providing critical intellectual content. AS and AC provided expertise in exercise oncology and methods of evaluation, contributing to the design of the exercise components of the intervention and providing critical intellectual content. DZHL provided clinical oversight and expertise. SL, LH, FW, MW and AB provided expertise in trial process and management. HM provided statistical expertise and devised the analysis plan with CG, SJ and MPWG. All authors contributed critically to the revision and final approval of the manuscript.

Funding Funding has been received from Macmillan Cancer Support (award/ grant number is not applicable), the Wessex Cancer Alliance (award/grant number is not applicable), the National Lottery (Digital, Culture Media and Sport grant number 20142065) and the Clinical Research Network (award/grant number is not applicable) to support this trial. This protocol includes independent work (CS) supported by the National Institute for Health Research (NIHR) Biomedical Research Centre at The Royal Marsden NHS Foundation Trust and the Institute of Cancer Research, London. MG is partly funded by the NIHR Senior Investigator Scheme and the NIHR Southampton Biomedical Research Centre. SL is supported by the National Institute for Health Research through the NIHR Southampton Biomedical Research.

Disclaimer The views expressed are those of the authors and not necessarily those of the NIHR or the Department of Health and Social Care.

Competing interests None declared.

Patient consent for publication Not required.

Provenance and peer review Not commissioned; externally peer reviewed.

Supplemental material This content has been supplied by the author(s). It has not been vetted by BMJ Publishing Group Limited (BMJ) and may not have been peer-reviewed. Any opinions or recommendations discussed are solely those of the author(s) and are not endorsed by BMJ. BMJ disclaims all liability and responsibility arising from any reliance placed on the content. Where the content includes any translated material, BMJ does not warrant the accuracy and reliability of the translations (including but not limited to local regulations, clinical guidelines, terminology, drug names and drug dosages), and is not responsible for any error and/or omissions arising from translation and adaptation or otherwise.

Open access This is an open access article distributed in accordance with the Creative Commons Attribution Non Commercial (CC BY-NC 4.0) license, which permits others to distribute, remix, adapt, build upon this work non-commercially, and license their derivative works on different terms, provided the original work is properly cited, appropriate credit is given, any changes made indicated, and the use is non-commercial. See: http://creativecommons.org/licenses/by-nc/4.0/.

ORCID iDs

Chloe Grimmett http://orcid.org/0000-0002-7540-7206

Andrew Scott http://orcid.org/0000-0003-1478-8962 


\section{REFERENCES}

1 Macmillan Cancer Support. The forgotten ' $C$ '? The impact of Covid-19 on cancer care, 2020.

2 Sud A, Jones ME, Broggio J. Collateral damage: the impact on cancer outcomes of the COVID-19 pandemic. medRxiv 2020.

3 Kuderer NM, Choueiri TK, Shah DP, et al. Clinical impact of COVID-19 on patients with cancer (CCC19): a cohort study. Lancet 2020;395:1907-18.

4 Jordan RE, Adab P, Cheng KK. Covid-19: risk factors for severe disease and death. BMJ 2020;368:m1198.

5 Silver JK. Prehabilitation could save lives in a pandemic. BMJ 2020;369:m1386.

6 Bruce J, Thornton AJ, Powell R, et al. Psychological, surgical, and sociodemographic predictors of pain outcomes after breast cancer surgery: a population-based cohort study. Pain 2014;155:232-43.

7 Foster C, Haviland J, Winter J, et al. Pre-Surgery depression and confidence to manage problems predict recovery trajectories of health and wellbeing in the first two years following colorectal cancer: results from the crew cohort study. PLoS One 2016;11:e0155434.

8 Mavros MN, Athanasiou S, Gkegkes ID, et al. Do psychological variables affect early surgical recovery? PLoS One 2011;6:e20306.

9 Macmillan Cancer Support. Coronavirus: half a million people with cancer are too scared to leave the house, 2020. Available: https:// news.sky.com/story/coronavirus-half-a-million-people-with-cancerare-too-scared-to-leave-the-house-research-shows-12017108

10 Trials.gov. The Wessex Fit-4-Cancer Surgery Trial (WesFit), 2018. Available: https://clinicaltrials.gov/ct2/show/NCT03509428

11 Macmillan Cancer Support, RCoA and NIHR. Principles and guidance for prehabilitation within the management and support of people with cancer, 2019.

12 Goode AD, Lawler SP, Brakenridge CL, et al. Telephone, print, and web-based interventions for physical activity, diet, and weight control among cancer survivors: a systematic review. J Cancer Surviv 2015;9:660-82

13 Turner RR, Steed L, Quirk H, et al. Interventions for promoting habitual exercise in people living with and beyond cancer. Cochrane Database Syst Rev 2018;9:Cd010192.

14 Grimmett C, Corbett T, Brunet J, et al. Systematic review and metaanalysis of maintenance of physical activity behaviour change in cancer survivors. Int J Behav Nutr Phys Act 2019;16:37.

15 McAuley E, Szabo A, Gothe N, et al. Self-Efficacy: implications for physical activity, function, and functional limitations in older adults. Am J Lifestyle Med 2011;5:361-9.

16 Aaronson NK, Ahmedzai S, Bergman B, et al. The European organization for research and treatment of cancer QLQ-C30: a quality-of-life instrument for use in international clinical trials in oncology. J Natl Cancer Inst 1993;85:365-76.

17 Herdman M, Gudex C, Lloyd A, et al. Development and preliminary testing of the new five-level version of EQ-5D (EQ-5D-5L). Qual Life Res 2011;20:1727-36.

18 Lorig KR, Sobel DS, Stewart AL, et al. Evidence suggesting that a chronic disease self-management program can improve health status while reducing hospitalization: a randomized trial. Med Care 1999;37:5-14.

19 Hibbard JH, Mahoney ER, Stockard J, et al. Development and testing of a short form of the patient activation measure. Health Serv Res 2005;40:1918-30.
20 Mitchell AJ, Baker-Glenn EA, Granger L, et al. Can the distress thermometer be improved by additional mood domains? Part I. initial validation of the emotion thermometers tool. Psychooncology 2010;19:125-33.

21 Mitchell AJ, Morgan JP, Petersen D, et al. Validation of simple visual-analogue thermometer screen for mood complications of cardiovascular disease: the emotion thermometers. J Affect Disord 2012;136:1257-63.

22 Weiss DS. The Impact of Event Scale: Revised. Cross-cultural assessment of psychological trauma and PTSD. In: International and cultural psychology. New York, NY, US: Springer Science + Business Media, 2007: 219-38.

23 Amireault S, Godin G, Lacombe J, et al. The use of the GodinShephard leisure-time physical activity questionnaire in oncology research: a systematic review. BMC Med Res Methodol 2015;15:60.

24 Godin GSR. Godin leisure-time exercise questionnaire. Medicine Science Sports and Exercise 1997;26:S36-8.

25 World Cancer Research Fund. Cancer health check. Available: https://www.wcrf-uk.org/uk/cancer-health-check

26 Jager-Wittenaar $\mathrm{H}$, de Bats HF, Welink-Lamberts BJ, et al. SelfCompletion of the Patient-Generated subjective global assessment short form is feasible and is associated with increased awareness on malnutrition risk in patients with head and neck cancer. Nutr Clin Pract 2020;35:353-62.

27 Ottery FD. Definition of standardized nutritional assessment and interventional pathways in oncology. Nutrition 1996;12:S15-19.

28 Alonso J, Permanyer-Miralda G, Cascant P, et al. Measuring functional status of chronic coronary patients. reliability, validity and responsiveness to clinical change of the reduced version of the Duke activity status index (DASI). Eur Heart $J$ 1997;18:414-9.

29 Bredin SSD, Gledhill N, Jamnik VK, et al. PAR-Q+ and ePARmed-X+: new risk stratification and physical activity clearance strategy for physicians and patients alike. Can Fam Physician 2013;59:273-7.

30 Warburton DER, Jamnik V, Bredin SSD, et al. 2020 physical activity readiness questionnaire for everyone $(P A R-Q+)$ and electronic physical activity readiness medical examination (ePARmed- $\mathrm{X}+$ ): 2020 PAR-Q+. The Health \& Fitness Journal of Canada 2019;12:58-61.

31 National Institute for Healtha and Care Excleence. Improving supportive and palliative care for adults with cancer, Cancer service guideline [CSG4, 2004.

32 Tinati T, Lawrence W, Ntani G, et al. Implementation of new Healthy Conversation Skills to support lifestyle changes - what helps and what hinders? Experiences of Sure Start Children's Centre staff. Health Soc Care Community 2012;20:430-7.

33 Michie S, Richardson M, Johnston M, et al. The behavior change technique taxonomy (V1) of 93 hierarchically clustered techniques: building an international consensus for the reporting of behavior change interventions. Ann Behav Med 2013;46:81-95.

34 May CR, Mair F, Finch T, et al. Development of a theory of implementation and integration: normalization process theory. Implement Sci 2009;4:29.

35 Giesinger JM, Loth FLC, Aaronson NK, et al. Thresholds for clinical importance were established to improve interpretation of the EORTC QLQ-C30 in clinical practice and research. J Clin Epidemiol 2020;118:1-8. 